Relations industrielles

Industrial Relations

\title{
Famille, industrialisation, logement, par Andrée Michel, Centre National de la Recherche Scientifique, 13 Quai Anatole France, Paris VII, 1959.
}

\section{Laurent Bélanger}

Volume 16, numéro 3, juillet 1961

URI : https://id.erudit.org/iderudit/1021782ar

DOI : https://doi.org/10.7202/1021782ar

Aller au sommaire du numéro

Éditeur(s)

Département des relations industrielles de l’Université Laval

ISSN

0034-379X (imprimé)

1703-8138 (numérique)

Découvrir la revue

Citer ce compte rendu

Bélanger, L. (1961). Compte rendu de [Famille, industrialisation, logement, par Andrée Michel, Centre National de la Recherche Scientifique, 13 Quai Anatole France, Paris VII, 1959.] Relations industrielles / Industrial Relations, 16(3), 363-363. https://doi.org/10.7202/1021782ar

Tous droits réservés (C Département des relations industrielles de l’Université Laval, 1961
Ce document est protégé par la loi sur le droit d'auteur. L'utilisation des services d’Érudit (y compris la reproduction) est assujettie à sa politique d'utilisation que vous pouvez consulter en ligne.

https://apropos.erudit.org/fr/usagers/politique-dutilisation/ 
lation qui restreigne indùment le droit de grève et amène l'intervention de l'Etat aux premiers stages d'un conflit. «L'intérêt public » est beaucoup moins souvent en cause qu'on ne le croit.

\section{L.}

Famille, industrialisation, logement, par Andrée Michel, Centre National de la Recherche Scientifique, 13 Quai Anatole France, Paris VII, 1959.

Pour ceux qui s'intéressent à la sociologie familiale, en particulier, aux modifications dans les structures et les systèmes de valeurs familiaux par suite de la révolution industrielle, voici un ouvrage élaboré sur le sujet.

L'étude porte sur 276 ménages habitant les hôtels meublés à Paris et dans la Seine. Un premier chapitre donne une description de l'habitat et des conditions économiques.

Les transformations au sein de la vie familiale pour s'ajuster au conditionnement externe du système industriel accusent des différences suivant les types de famille: industrielle française, semipatriarchale espagnole, patriarchale algérienne. La structure économique d'un cadre rural archaïque et la structure juridique établissaient un mode de transmission de la propriété dans la famille ancienne patriarchale. Les membres tiraient leur identité du groupe familial. Chacun avait un statut et un rôle fixes. La hiérarchie consacrait la supériorité des parents sur les enfants, celle des sexes fut présentée comme de droit divin, la morale sexuelle était respectée, le mariage était considéré comme un sacrement, les liens de parenté s'étendaient presque à l'infini, la sécurité était assurée totalement par la famille ou le voisinage. Dans l'ensemble, l'organisation interne de la famille était basée sur la hiérarchie, le conformisme et la répression. La famille industrielle présente un modèle différent. Elle tire son mode d'existence du salariat, possède peu de biens. La tendance à l'égalitarisme règne dans les relations entre époux, entre parents et enfants. L'infériorité de la femme disparaît si on considère le rôle qu'elle joue dans l'administration des biens. La liberté de choix du conjoint pour les jeunes, les loisirs à l'extérieur du ménage, l'interchangeabilité des rôles entre l'homme et la femme diminuent l'autorité du «chef de famille ». On considère les institutions (mariage, religion) comme étant au service de la personne et non l'inverse.

Des transformations économiques accompagnent le passage de la famille ancienne à la famille conjugale: « partage par la famille de ses rôles traditionnels avec des tiers (organismes de sécurité sociale, de loisirs) indépendance de la personne par rapport à un rôle et un statut préétablis et fixes, tendance à l'identification sociale avec un rôle extérieur au groupe familial, concurrence des valeurs économiques ». Cette évolution enlève le caractère sacré aux rites relatifs à la naissance (idéal de la famille prolifique), à l'adolescence, au mariage et à la mort.

Au cours de l'adaptation de la vie familiale aux conditionnements externes engendrés par la guerre et la révolution industrielle, une conception de la famille ancienne a survécu. Elle diffère cependant selon le type de famille étudié. L’auteur signale un « retard culturel » qui consiste en la « dysharmonie entre les fonctions économiques de la famille d'aujourd'hui et l'idée ancienne que l'on s'en fait encore. Cette étude amène l'auteur à préciser le concept de «famille». \& Dans sa forme la plus complète, la famille conjugale est constituée par le père, la mère, les enfants, mais elle peut aussi se reduire au couple sans enfants, à un adulte ayant un ou plusieurs enfants.

Dans la conclusion, l'auteur met en exergue les facteurs qui paraissent agir le plus sur la structure de la famille:

a) la structure de la société globale (développement technologique, division du travail, mode de propriété).

b) l'intégration des familles aux classes sociales ou aux multiples groupements.

c) le logement.

LAURent Bélanger

European Coal Mining Union: Structure and Function, by Frederic Meyers, Institute of Industrial Relations, University of California, Los Angeles, 1961. 